\title{
LA-UR-21-27803
}

Approved for public release; distribution is unlimited.

Title: $\quad$ End of Summer Presentation

Author(s): $\quad$ Martinez Sanchez, Diego

Intended for: $\quad$ To keep in my academic portfolio

Issued: 2021-08-04 
Disclaimer:

Los Alamos National Laboratory, an affirmative action/equal opportunity employer, is operated by Triad National Security, LLC for the National Nuclear Security Administration of U.S. Department of Energy under contract 89233218CNA000001. By approving this article, the publisher recognizes that the U.S. Government retains nonexclusive, royalty-free license to publish or reproduce the published form of this contribution, or to allow others to do so, for U.S. Government purposes. Los Alamos National Laboratory requests that the publisher identify this article as work performed under the auspices of the U.S. Department of Energy. Los Alamos National Laboratory strongly supports academic freedom and a researcher's right to publish; as an institution, however, the Laboratory does not endorse the viewpoint of a publication or guarantee its technical correctness. 


\section{End of Summer Presentation Summer 2021}

Diego Martinez August $4^{\text {th }}$ 


\section{Overview}

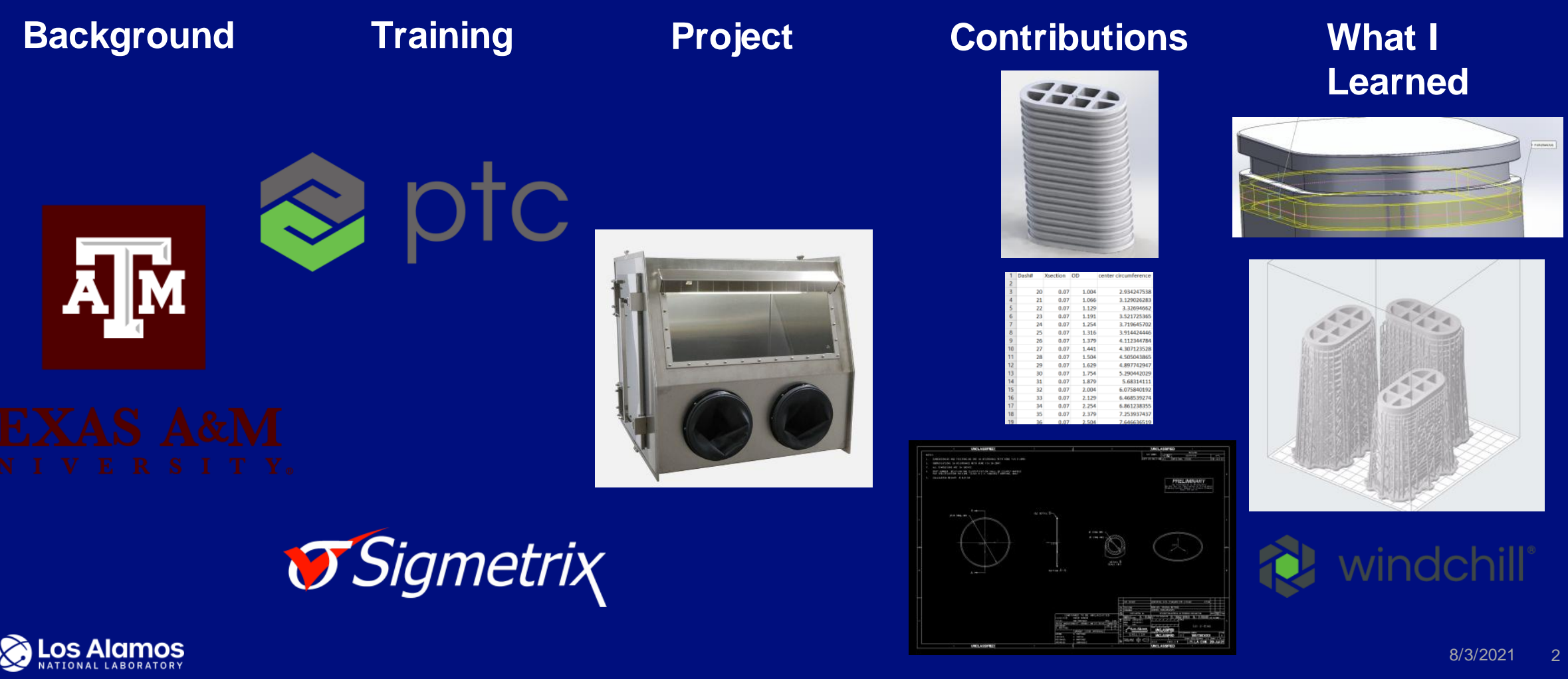




\section{Background}

- Originally from Spain

- Lived in England and Mexico

- High school in Houston, Texas
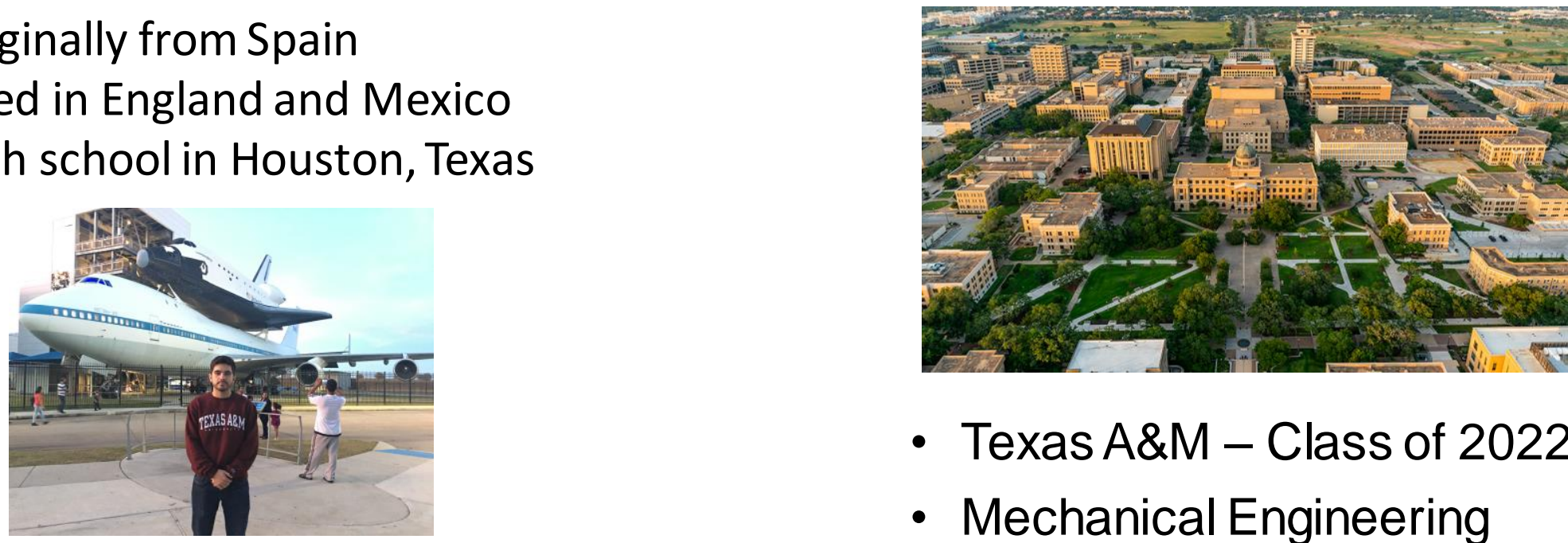

- Texas A\&M - Class of 2022

- Mechanical Engineering

- Interests:

- Astrophysics

- Drone cinematography 


\section{Training}

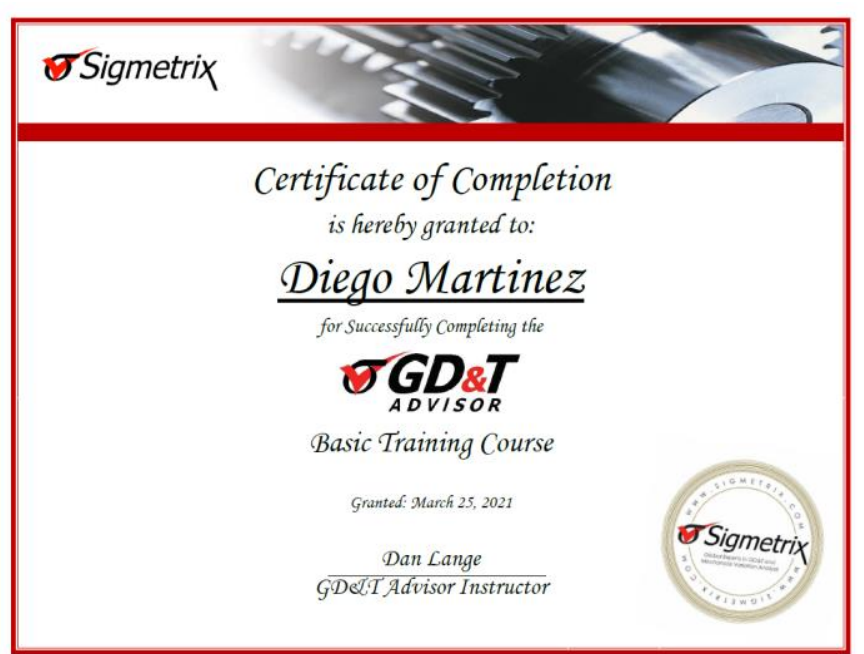

GD\&T Levels 1,2 and Advisor

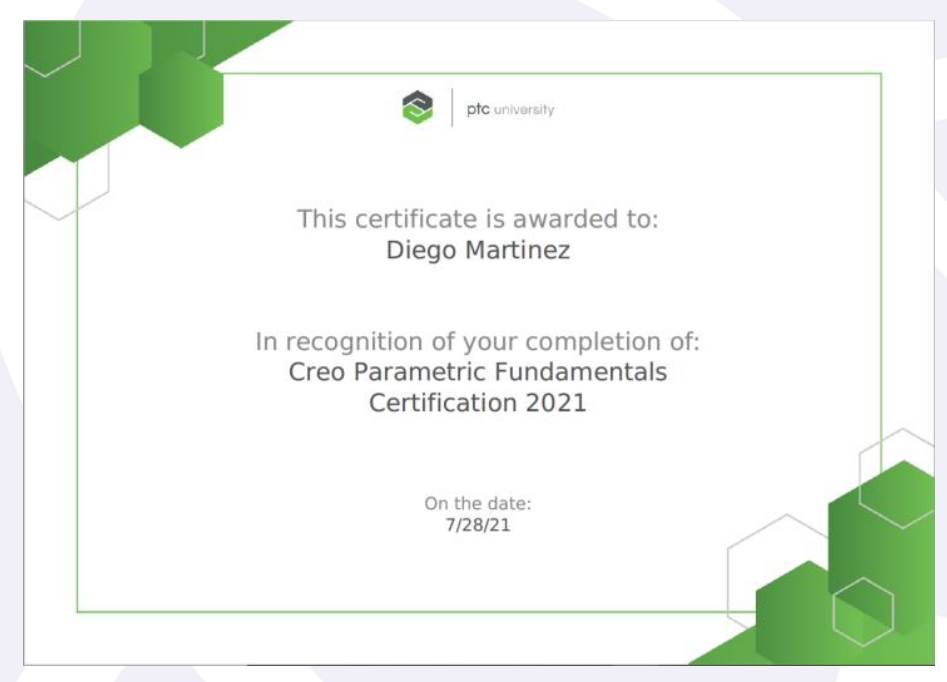

Creo Parametric Fundamentals 


\section{Project}

Decontaminating Gloveboxes
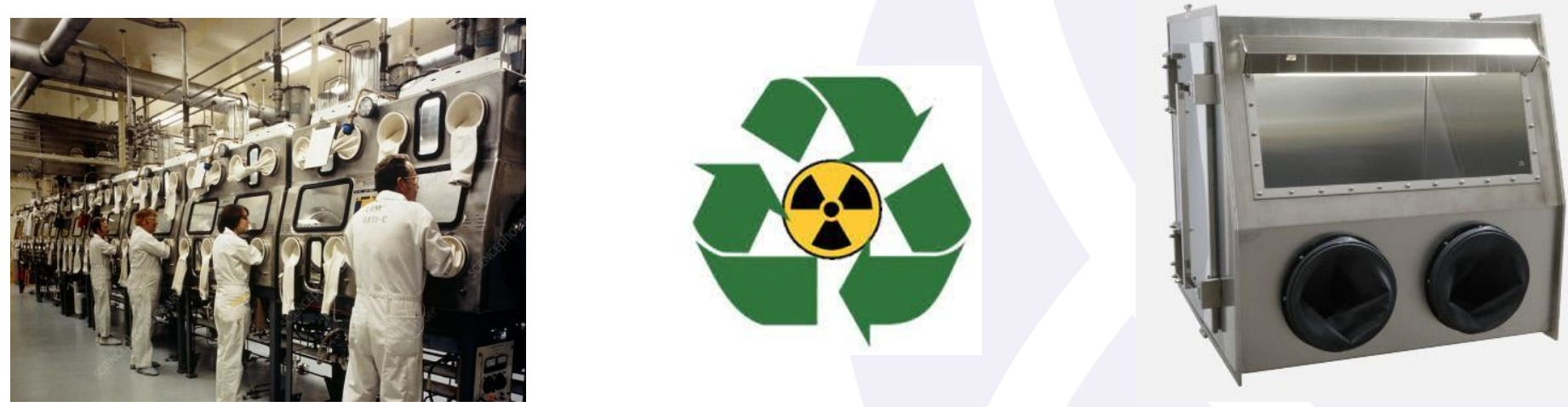


\section{Project}

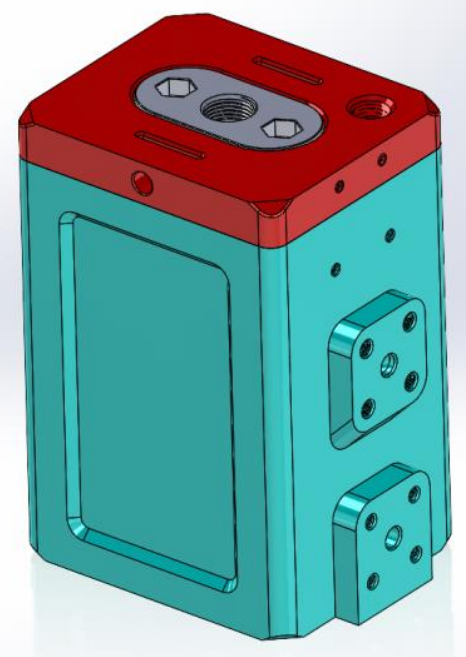

[Electrolytic] Flowcell

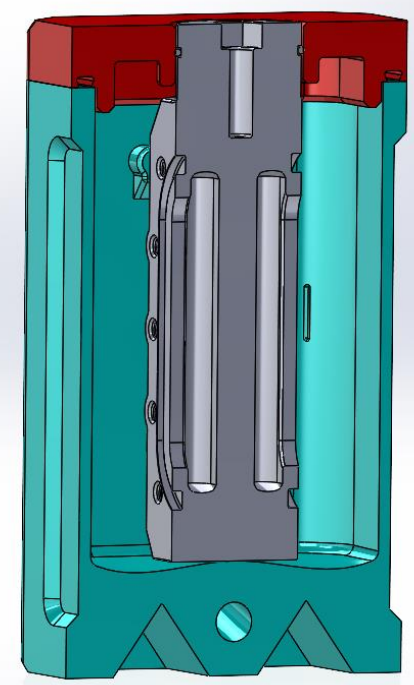

Cathode/Anode

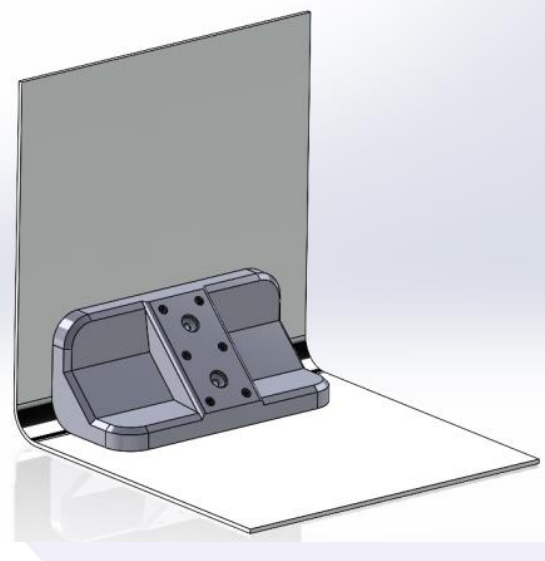

Edge Vacuum Head 


\section{Contributions}

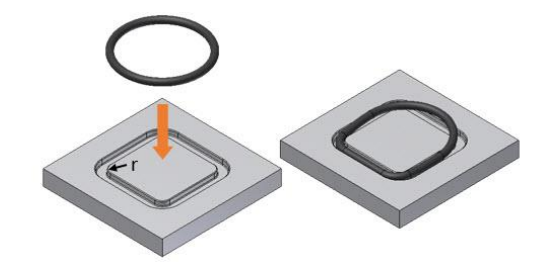

Non-circular o-ring groove

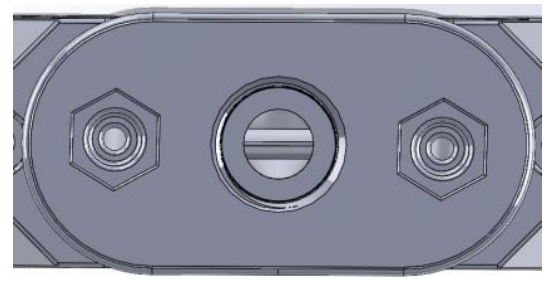

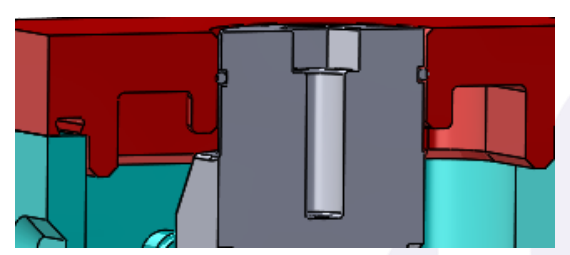

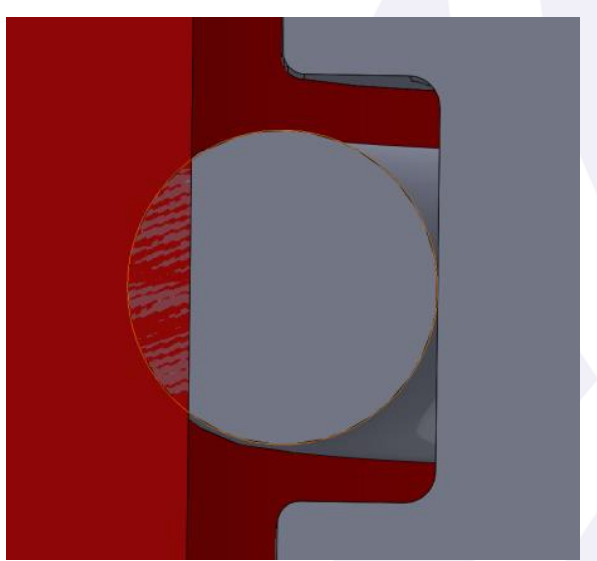

Overlap/Squeeze
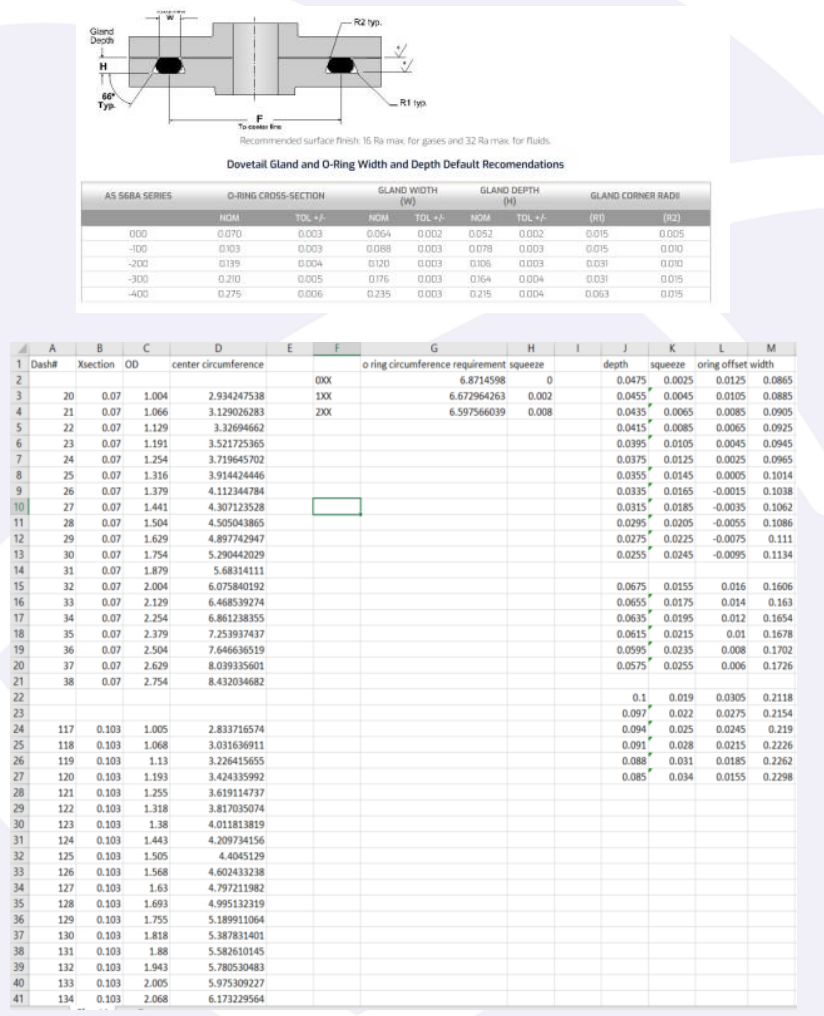


\section{Contributions}

Testing multiple grooves on a single part

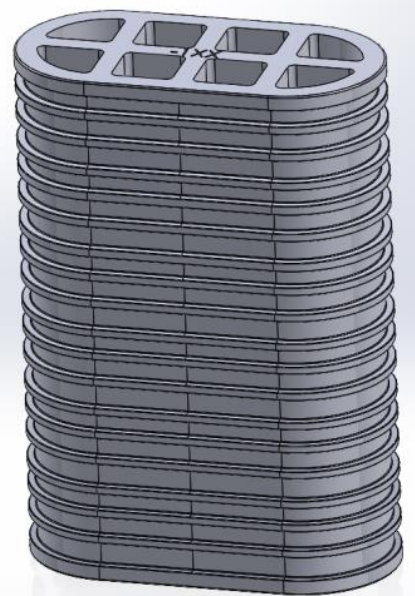

3D printing prototypes
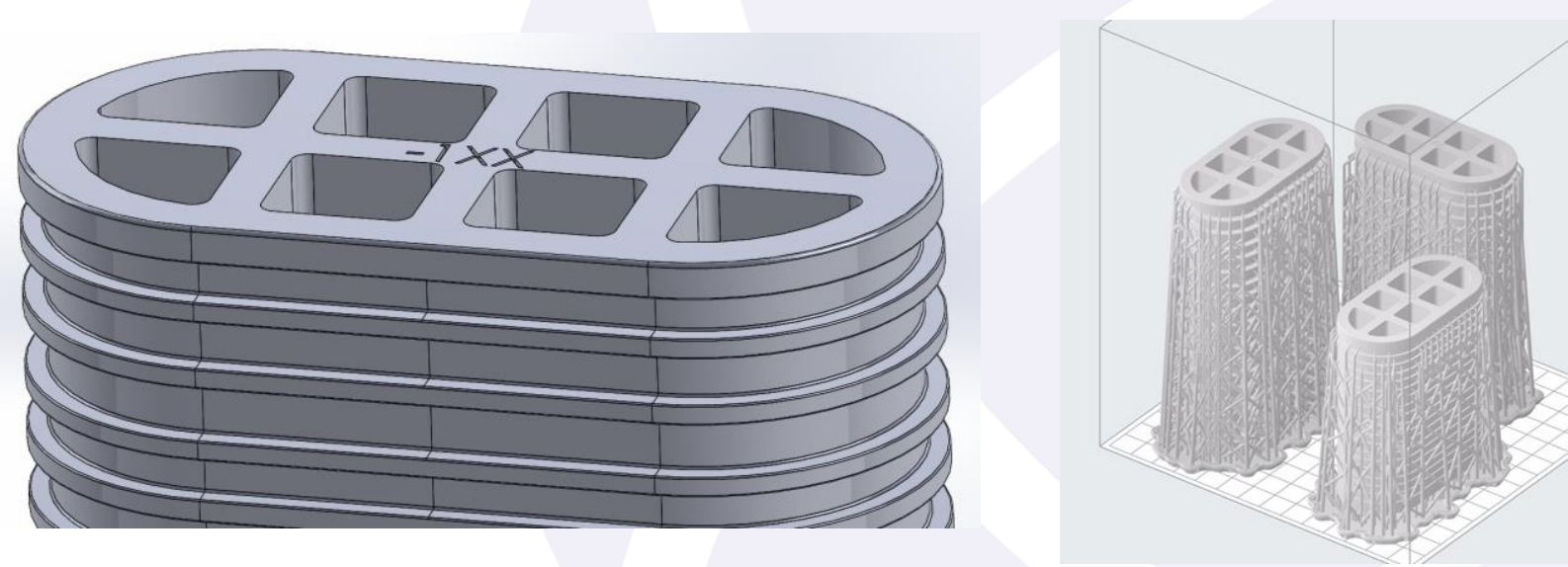


\section{Contributions}

90 degree airtight surface
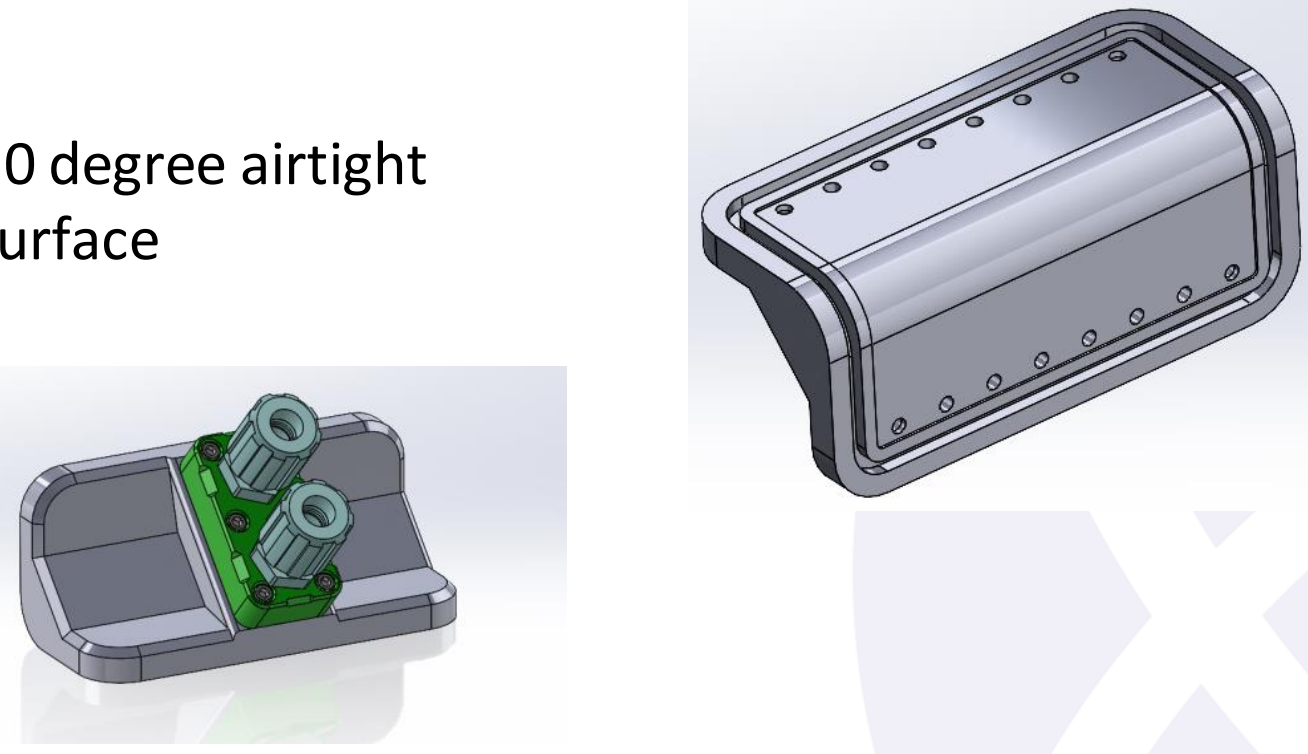

Dovetail groove

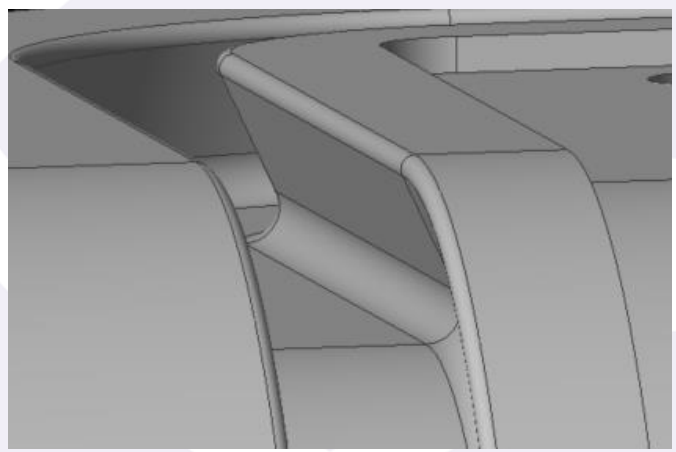




\section{Contributions}

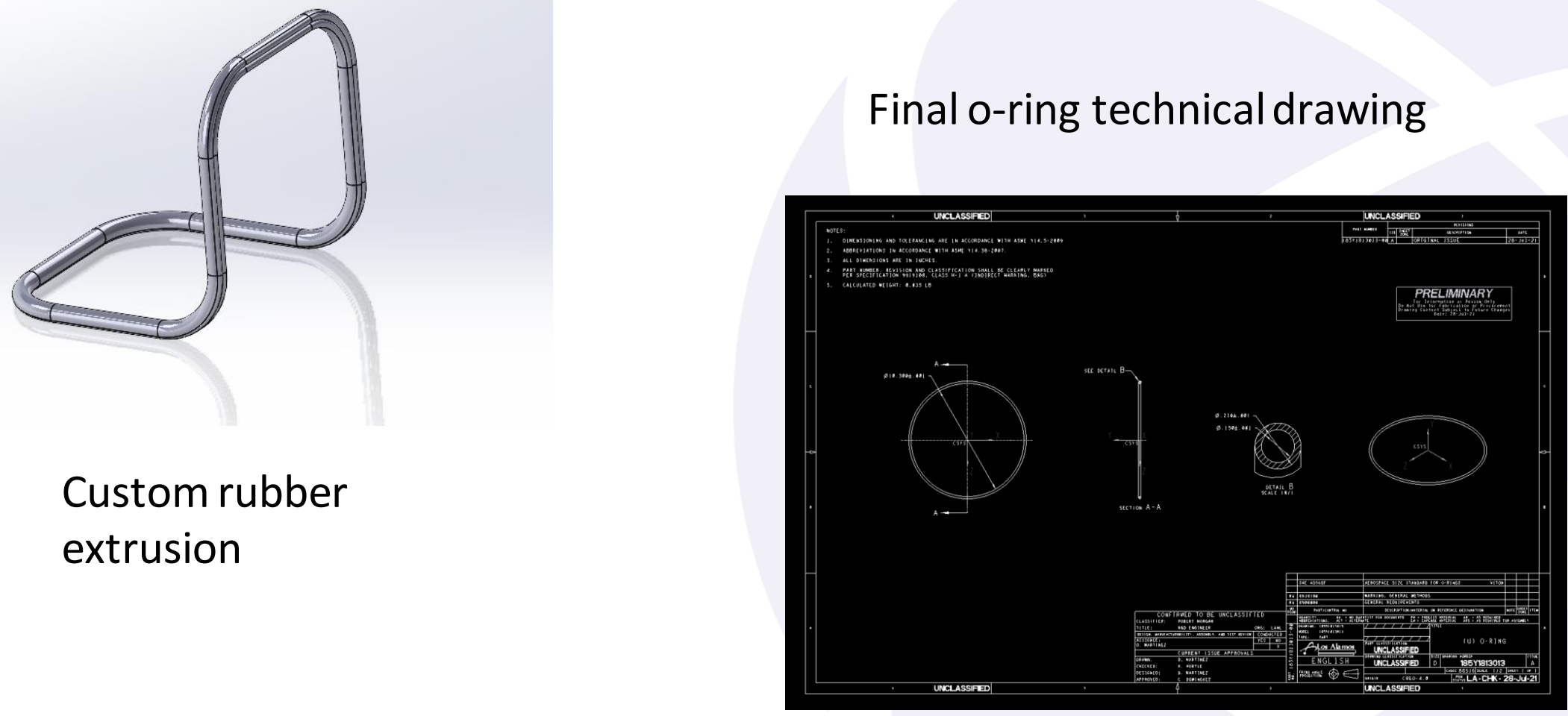




\section{What I learned}

- Swept Cut

- Technical Standards

- Excel for CAD

- Manufacturing

- O-Ring Sealing

- Design Meetings

- W-11 Drawing/Windchill
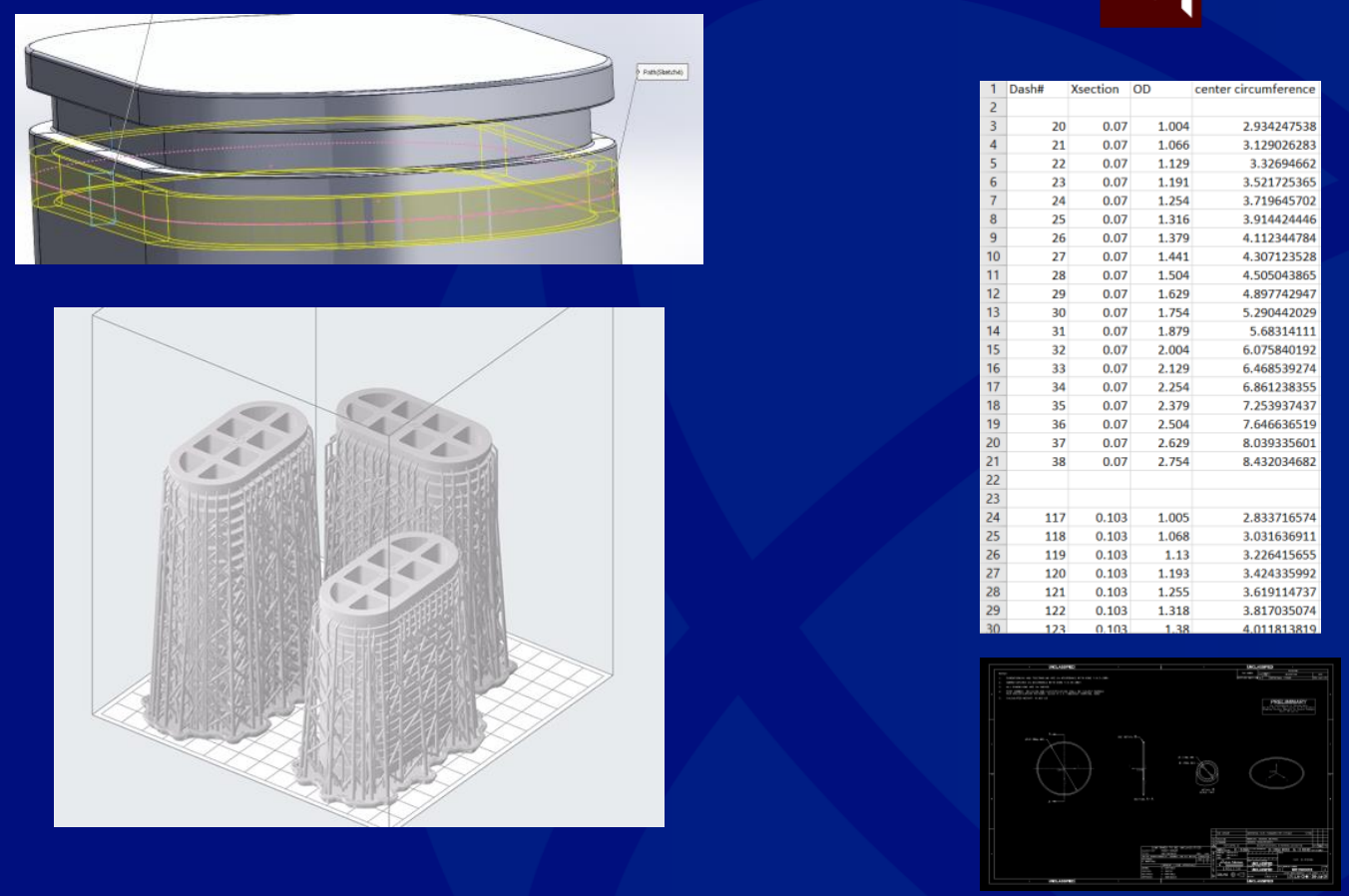